\title{
The Simple Method to Process Intersecting Line with Macro Program
}

\author{
Mingqing Wang \\ Beijing Information Technology college, No.90 XinZhuang, Hua Xiang, Feng Tai District, Beijing, China \\ wangmq@bitc.edu.cn
}

\begin{abstract}
Intersecting line usually appears on the 3-way or 4- way interface spare parts, forming the curves of surfaces. During the course of processing by CNC machine, the CNC processing program established by using computer assistant programming will greatly improve the effectiveness of programming. However, the program worked out in this way need a huge amount of data. It is difficult to dig out the root reason of problem and to fix the problem. With the help of Macro program, give full play to the function of CNC system and the craft and experience of programmer, it will be easy to write out simple, easy understanding, flexible and easy using program.
\end{abstract}

Keywords-CNC processing; Intersecting Line; Macroprogram; Node

By CAD, the program generated by software can be recognized to process spare parts. This automatically generated software is almost the composition of simple order of lines and arc. Though the data is accurate, but very complex and hardly to be understood. However, manual programming is that a programmer writes spare parts processing program directly using order from machine. Manual programming will make full use of the function of CNC system, the craft and experience of processing of programmer and write out simple, easy understanding, flexible and friendly program.

To make processing program simple, easy understanding, flexible and easy using, it is necessary to make full use of the function of machine, parameter and macro program to write program. CNC system equipped customers with powerful macro program similar to high-level language. Customers can use variable to conduct arithmetic operation, logical operation and function operation, in addition, macro-program also provide loop statement, branched statement and subroutine call statement, which is good to write kinds of complex spare parts processing program, reducing, even eliminating boring value calculating and simplifying the quantity of program in manually writing program. Using variable in program, by evaluating and adjusting variable in program, equip the program with special function. A program with such variable is called Macro-program. Many programming skill is applied in macro-program, such as the establishing of mathematical model, the express of mathematical relations, the choose of processing cutters, the gain and loss to the way of the cutter moving, etc. with all these functions, macro program can make spare parts with high precision, especially for those parts with averaged level of difficulty, which enable to write program using macro program faster than that using auto programming. Sometimes the length of program is dozens of times or hundreds of time of macro program, and even more and the time spent on processing will also be greatly increased.

The three axis in CNC are shown as three processing surfaces, with Code $G$ as prefix of surfaces, Surface G17XY, Surface G18 XY, Surface G19YZ.

Take the intersecting line processing as an example, to demonstrate the flexible application of macro program.Intersecting lines usually appears on the intersection spare parts, such as three-way and four-way, etc. forming the curves of surfaces. In the course of processing a spare part, the normal working procedure is that a miller produces the external shape of a spare part with required margin. Then a lathe operator makes out interface screw thread, through-hole, etc. The arc surfaces at the intersection of spare parts need to be completed on CNC miller.

Fig. 1 The spare part is intersected by two cylinders with different size, which has typical intersection character. After milling, lathing, the spare part is changed to the shape as the external shape of Fig 2. Further processing is required to achieve the required external shape of the spare part.

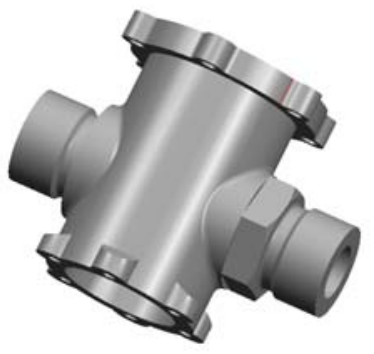

Figure 1. Completed Spare Part 


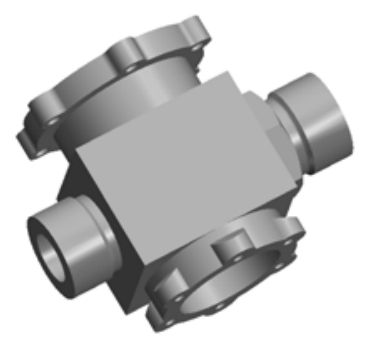

Figure 2. Ready to be Processed after Milling and Lathing

Spare part Analysis:

The area that need to be processed is intersected by two cylinders, and the intersecting lines are composed of intersection of round section that has been divided. These intersections are the required programming node. The node coordinates can be worked out by mathematical formula. Through parameter calculating, work out several sections with several intersections of sections, i.e. programming nodes. Then get the coordinate of nodes through calculating, write out contour program one by one. These contour of section form the intersection surfaces.

Processing Analysis:

The surface of spare parts that need to be processed is three-dimension surface. Three-dimension processing usually use software to write program. Software programming needs huge quantity of data with poor generality. The following work will be very tedious. Re-editing, correcting have to be done in different processing coordinate system. Manual programming will make use of the characteristic of machines, simplify the programming by dividing spare parts and processing one by one.

The prime aspect need to be considered is the clamping of the spare parts and the building of processing coordinate system. This spare parts need to be clamped by plier, the two big ends cling closely to mouth of plier. The small end is paralleled to the top of working desk. The arrow pointing direction is the $\mathrm{X}$ forward direction of spare part. Using ball-end mill cutter to perform scanning like depth processing. In programming, the direction $\mathrm{Y}$ is the direction of layering. The initial value of parameter no. 1 is the small arc plus radius of the cutter. No 5 is the bigger arc plus radius of cutter. No 6 is small arc plus radius of cutter, No. 10 is the initial value of the layers. No. 8 is the quantity of layers. The program is as follow. $\# 5=22$

(Radius of the big arc)

\author{
\#6=17 (Radius of the small arc) \\ $\# 7=25$ \\ $\# 8=0.25 \quad$ (Variable value ) \\ $\# 10=0$ (Initial value of the \\ G18 \\ (The surface for \\ processing ) \\ $\# 1=-17$ \\ WHILE[\#1LE17]DO1 \\ $\# 9=\# 6-\# 10$ \\ $\# 2=S Q R T[[\# 6 * \# 6]-[\# 9 * \# 9]] \quad($ Value of Z $)$ \\ $\# 3=$ SQRT[[\#5*\#5]-[\#2*\#2]] (Value of big arc on the \\ direction of $\mathrm{X}$ ) \\ G0Y\#1X\#7 \\ G1Z\#2F300 \\ $\mathrm{X \# 3}$ \\ G02X-\#3R\#5 \\ G1X-\#7 \\ G0Z25 \\ $\# 1=\# 1+\# 8$ \\ $\# 10=\# 10+\# 8$ \\ END1 \\ G0Z50M9 \\ X300Y0 \\ M30
}

depth )

By CAD, the data volume of program that worked out by means of software are only tens of $k b$, or hundreds $k b$ at most. However the data volume of manual programming is normally less than $1 \mathrm{~kb}$. This fully demonstrate the powerful function of macro program, the flexibility, high effective ness, swift of Macro program, and it plays an important part in addition to processing programming. By making full use of the regularity of spare parts, developing the calculating capability of CNC system to simplify programming and improve the effectiveness.

\section{REFERENCES}

[1] WANG X iao-peng: AutomaticGeneration of $G$ Code in Numerically ControlledMachining for Perpendicular Intersecting Line Type Notch Making (MECHANICAL ENGINEERING \& AUTOMATION; N o. 4Aug.2006)

[2] TAN Xiao: Strip-width Maximization Technologyfor Machining Circular Blending Surfaces with Torus Cutter (MECHANICAL ENGINEERING \& AUTOMATION ;N o. 1 Feb.2014)

[3] XUE Zhi-yong: The Automatic Programming of Intersecting Line Processing (Science and Technology Consulting Herald; N o. 27.2007) 